\title{
A Real-Time Implementable Model-Predictive Cruise Controller for Electric Vehicles and Energy-Efficient Driving
}

\author{
Tim Schwickart ${ }^{1}$, Holger $\operatorname{Voos}^{1}$ and Mohamed Darouach ${ }^{2}$
}

\begin{abstract}
This paper presents a novel energy-efficient model-predictive cruise control formulation for electric vehicles. The controller and the underlying dynamic model are designed to meet the properties of a series-production electric vehicle whose characteristics are identified by measurements. A predictive eco-cruise controller involves the minimisation of a compromise between terms related to driving speed and energy consumption which are in general both described by nonlinear differential equations. In this work, a coordinate transformation is used which leads to a linear differential motion equation without loss of information. The energy consumption map is approximated by the maximum of a set of linear functions which is implicitly determined in the optimisation problem. The reformulations finally lead to a model-predictive control approach with quadratic cost function, linear prediction model and linear constraints that corresponds to a piecewise linear system behaviour and allows a fast real-time implementation with guaranteed convergence. Simulation results of the MPC controller in closed-loop operation finally show the effectiveness of the approach.
\end{abstract}

\section{INTRODUCTION}

The cruising range is one of the most decisive drawbacks of electric vehicles and an important problem that needs to be solved in electric mobility. Since the on board (tank-towheel) efficiency of electric vehicles can hardly be improved, there are only two possibilities to increase the range. The first one is the improvement of the battery technology towards higher capacities and lower weights. The second opportunity is to address the driving style that has a huge influence on the energy consumption of a vehicle [1]. Due to possible savings of 10 to $20 \%$, it is a promising approach to improve the driving style in order to save energy.

A sophisticated way to address this problem is controlling the driving speed automatically by a driver-assistance system (eco-cruise control). Eco-cruise control can be described as an optimal control problem [2], [3]. The accelerator and brake pedal positions are the control inputs of the system while the driving speed and the energy consumption are given by an underlying dynamic vehicle model (based on the previous knowledge of the speed limits and the road slope). The control inputs are the optimisation variables that minimise a cost function containing terms related to driving speed and energy consumption. As the car is running under changing traffic and environment conditions, it is hardly possible to calculate and apply the complete optimal driving

\footnotetext{
${ }^{1}$ Tim Schwickart and Holger Voos are with the Interdisciplinary Centre for Security, Reliabilty and Trust (SnT), University of Luxembourg, Luxembourg

${ }^{2}$ Mohamed Darouach is with the Centre de la Recherche en Automatique de Nancy (CRAN), Université de Lorraine, France
}

strategy in advance. A suitable approach to overcome this problem is to apply model-predictive control (MPC) in a receding horizon fashion, where the optimisation is carried out for a finite prediction horizon and is repeated at every time step. This control strategy has been considered as the tool of choice for the eco-cruise control of fuel-powered cars in several works [3], [4], [5], [6]. Recently, eco-cruise control for purely electric vehicles has been considered in [7], [8]. The biggest challenge in the application of MPC is the requirement of a fast online-optimisation which is hampering a real-time implementation. Therefore, the formulation of the optimal control problem is decisively important in order to achieve a fast solution. The most desirable formulation comprises a quadratic cost function and linear constraints including a linear dynamic plant model, since efficient solvers with guaranteed convergence are available for the resulting discretised quadratic optimisation problem.

However, an overall linearisation of the vehicle dynamics around one operation point is not satisfactory since the prediction has to be carried out over a wide range of operating points whereas a linearisation only yields good results in the area close to the operating point. Previous works use analytical solutions of the nonlinear optimal control problem based on Pontryagin's Maximum Principle (indirect methods) [2], [3] or alternatively efficient discretisation techniques to solve the nonlinear optimisation problem directly [5], [9], [6]. Using analytical solutions however, the optimal controller cannot be designed in a flexible way since no constraints on the state variables, dynamically changing weightings or measured disturbances can be considered. On the other hand, the numerical methods for nonlinear optimisation do not guarantee a (fast) convergence of the optimisation algorithm.

This paper contributes a model-predictive eco-cruise controller especially for an electric car using a quadratic cost function and linear constraints. The linear dynamic model is obtained by reformulations of the equations and an exploitation of the optimisation problem setup instead of an overall linearisation (Section III). Thus, the nonlinearities are considered implicitly by the control system while the results are equivalent to a nonlinear approach. The proposed controller is simulated in closed-loop operation with a simulation model of a series electric vehicle - a Smart Electric Drive (ED) to investigate the closed-loop performance (Section IV).

\section{Overall System SetuP}

The overall system is planned to work as a driverassistance system mainly in highway and overland driving. It shall control the speed automatically depending on the 
road curvature, the road slope angle, the speed limits and the distance to the preceding vehicle.

A reference generator (not considered in this work) generates the speed set-point trajectory with respect to speed limits, the distance to the preceding car and the road curvature. Given this information, the model-predictive cruise controller aims at finding a traction force trajectory leading to an optimal compromise between speed reference tracking and minimisation of the energy consumption. Since the traction force cannot serve directly as control input to the vehicle, a subsidiary controller regulates the traction force by actuating the accelerator pedal. The brake pedal is not planned to be actuated in this setup. This paper focuses on the design of the MPC controller.

\section{Controller Design}

The synthesis of the energy-efficient model-predictive cruise controller comprises the underlying dynamical model as well as the constraints and the cost function.

\section{A. Underlying Dynamic Model for the Controller Design}

A suitable model needs to meet the dynamic behaviour of the Smart ED whose centre-piece is a permanent-magnet synchronous machine. This three-phase AC machine is able to work as motor or generator allowing energy recovery when decelerating. A lithium-ion battery serves as accumulator and supplies the synchronous machine via a DC/AC converter. The rear wheels are driven by the motor through a gear box with one fixed transmission ratio.

The model is subdivided into a model of the driving speed $v$ (Section III-A.1) and a model of the energy consumption $E_{e l}$ of the vehicle (Section III-A.2). The model input (and control input) of the MPC is the traction force at the wheels $F_{\text {trac }}$.

1) Model of the Longitudinal Motion Dynamics: The common approach to model the longitudinal dynamics of a vehicle is to consider the car as a point mass and describe a one-dimensional motion based on Newton's second law $\sum F=m \cdot \frac{d v}{d t}$. The main forces acting in longitudinal direction on the vehicle are the traction force $F_{\text {trac }}$ as well as the driving resistance forces [10].

The rolling resistance force $F_{r}$ is a function of the road slope angle $\alpha_{s l}$. The parameters are the vehicle kerb weight $m_{v}$, the payload $m_{l}$, the gravitational constant $g$ and the rolling resistance coefficient of the tyres $c_{r}$ [10].

$$
F_{r}=\left(m_{v}+m_{l}\right) \cdot g \cdot c_{r} \cdot \cos \left(\alpha_{s l}(t)\right)
$$

The grade resistance force $F_{g r}$ depends on road slope angle as well [10].

$$
F_{g r}=\left(m_{v}+m_{l}\right) \cdot g \cdot \sin \left(\alpha_{s l}(t)\right)
$$

The air drag resistance force $F_{d}$ is a function of the square of the driving speed $v$. The coefficients are related to the shape of the vehicle (projected front surface area $A_{v}$, air drag coefficient $c_{d}$ ) and the air density $\rho_{a}[10]$.

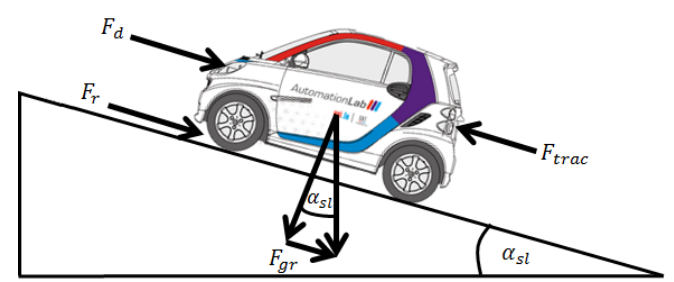

Fig. 1. Forces acting in longitudinal direction on the vehicle.

$$
F_{d}=\frac{1}{2} \cdot \rho_{a} \cdot c_{d} \cdot A_{v} \cdot v(t)^{2}
$$

A diagram of the forces acting in longitudinal direction is given in Fig. 1.

Given these forces, the acceleration of the vehicle in longitudinal direction can be computed from the difference between the traction force and the driving resistance forces divided by the equivalent mass of the vehicle $m_{e q}$.

$$
\begin{aligned}
\frac{d v(t)}{d t}= & \left(F_{\text {trac }}(t)-F_{r}\left(\alpha_{s l}(t)\right)\right. \\
& \left.-F_{g r}\left(\alpha_{s l}(t)\right)-F_{d}(v(t))\right) / m_{e q}
\end{aligned}
$$

The equivalent mass $m_{e q}$ is given by the relation $m_{e q}=$ $\left(m_{v}+m_{l}\right) \cdot e_{i}$ which takes the inertia of the drive train components into account by augmenting the vehicle mass $\left(m_{v}+m_{l}\right)$ by the constant factor $e_{i}$.

The vehicle specific parameters in (1) to (4) are accessible from data sheets [11]. The rolling resistance coefficient $c_{r}$ is assumed to be 0.01 . The gravity constant is assumed to be $g=9.81 \frac{\mathrm{m}}{\mathrm{s}^{2}}$ and the density of surrounding air to be $\rho_{a}=1.2 \frac{\mathrm{kg}}{\mathrm{m}^{3}}$. All parameters are summarised in Tab. I.

For the application of a predictive cruise controller however, it is useful to describe the model (4) as a function of the position $s$ instead of time, since the inputs related to the road ahead (slope angle and speed limits) are also given as functions of the position. The model can be reformulated by applying the following transformation:

$$
\frac{d}{d s}=\frac{d}{d t} \cdot \frac{d t}{d s}=\frac{d}{d t} \cdot \frac{1}{v}
$$

The reformulation (5) consequently leads to a motion equation depending on the inverse of a state variable (the velocity $v$ ). This is disadvantageous for a fast solution of the optimisation problem. Following the idea in related works ([6], [12]), the following coordinate transformation is applied to calculate the kinetic energy

$$
e_{k i n}=\frac{1}{2} \cdot m_{e q} \cdot v(t)^{2}
$$

of the moving vehicle instead of the driving speed. Since only positive speed values are considered, the speed can be calculated from the kinetic energy values at a given vehicle mass after the optimisation. Derivation of (6) with respect to position $s$ yields

$$
\frac{d e_{k i n}}{d s}=m_{e q} \cdot \frac{d v}{d t}
$$




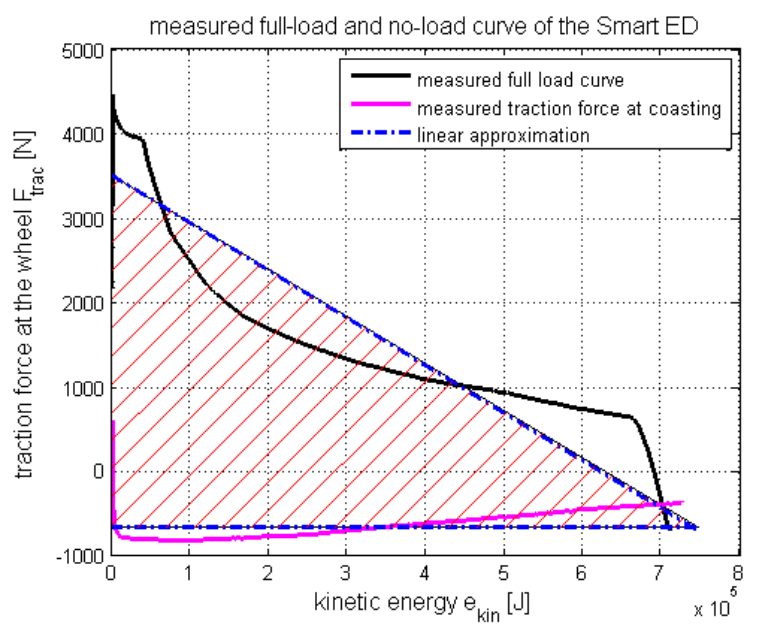

Fig. 2. Measured traction force of the Smart ED at full-load (100\% accelerator position, black line) and coasting (pedals released, pink line). The linear approximation is given in blue. The hatched area is the feasible region of the traction force $F_{t r a c}$.

By applying the coordinate transformation (7) to the motion equation (4), the following linear differential equation is obtained. The values of the sine and cosine functions of the road slope angle $\alpha_{s l}$ are assumed to be known and provided as a measurable disturbance.

$\frac{d e_{k i n}}{d s}=F_{t r a c}(s)-F_{r}\left(\alpha_{s l}(s)\right)-F_{g r}\left(\alpha_{s l}(s)\right)-F_{d}\left(e_{k i n}(s)\right)$

Herein, $F_{d}$ is rewritten in terms of kinetic energy:

$$
F_{d}\left(e_{k i n}\right)=\frac{1}{m_{e q}} \cdot \rho_{a} \cdot c_{d} \cdot A_{v} \cdot e_{k i n}(s)
$$

Since (8) is only valid for positive kinetic energy values, the inequality constraint

$$
e_{k i n} \geq 0
$$

must be imposed on the optimisation problem.

To stay within the limitations of the vehicle, the traction force needs to be limited. The measured full-load curve as well as the traction force at coasting (giving the maximum and minimum possible traction force depending on the kinetic energy of the moving vehicle) of the Smart Electric Drive are given in Fig. 2. The full-load curve has been measured at fully pushing the accelerator pedal but without pressing the "kick-down" switch below the accelerator pedal. The traction force at coasting has been measured with released pedals (slight energy recovery).

However, since only linear constraints should be considered here, the measured curves are linearised using a leastsquares approximation, resulting in the hatched polygon in Fig. 2 and represented by the following linear inequality:

$$
F_{t r, \min } \leq F_{\text {trac }} \leq c_{1} \cdot e_{k i n}+c_{2} ; \quad e_{k i n} \geq 0
$$

2) Dynamic Model of the Energy Consumption: In order to relate the electrical input of the drive train with the

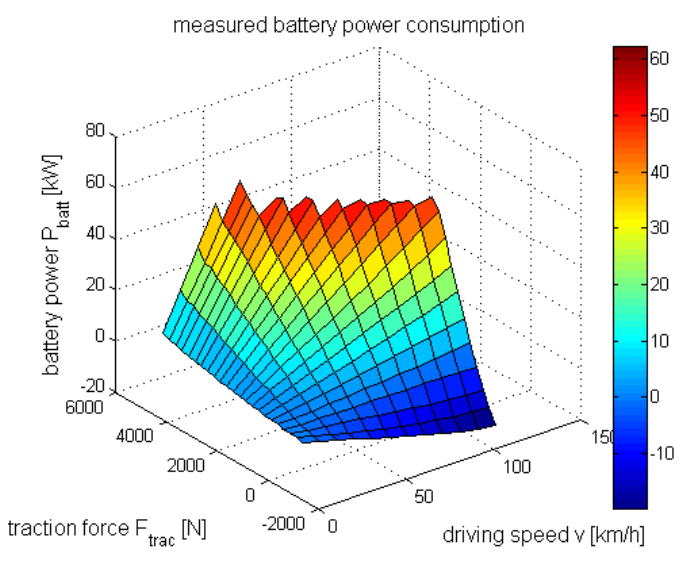

Fig. 3. Electrical battery power consumption as a function of the driving speed $v$ and the traction force $F_{t r a c}$

mechanical power output, the drive train and motor characteristics must be modeled. A detailed model of all physical processes in these components is not suitable here due to its complexity.

In this work, measurements of the overall drive train characteristics of the vehicle in quasi-static operation are available in the form of a characteristic map. The battery power $\left(P_{b a t t}=U_{\text {batt }} \cdot I_{\text {batt }}\right)$ is assumed to be a function of the traction force at the wheels $F_{\text {trac }}$ and the driving speed $v$. The data to set up this relation has been extracted from measurements in static operating points on an industrial dynamometer test bench. The resulting interpolated characteristics are depicted in Fig. 3. It can be assumed that these characteristics measured in quasi-static operation also hold in dynamic operation since the electrical time constants are much faster than the ones related to the mechanics. Quasistatic drive train models are widely used in applications with accurate results [10].

Since the vehicle motion model (8) is formulated with respect to the position $s$, the energy consumption must also be derived in terms of position. Here, it is advantageous that every operating point in the power consumption map (Fig. 3) is related to a certain driving speed due to the fixed transmission ratio of the vehicle. Hence, each point of the power consumption map is divided by its related driving speed $v$ according to reformulation (5) to obtain the energy consumption per meter. In addition to this, the $\mathrm{x}$ axis is rescaled in terms of the kinetic energy of the moving vehicle in order to fully comply with the reformulated motion equation (8). The resulting map of the energy consumption per meter as a function of the kinetic energy and the traction force is given in Fig. 4a. The objective is to implement an approximation of these characteristics in the underlying dynamic model of the controller.

One possible method for the approximation of energy consumption maps is the use of fitted polynomials [13]. Nevertheless, a closer look at Fig. 4a shows that the given characteristics are more suitable for a piecewise linear approximation since they can hardly be captured by one 


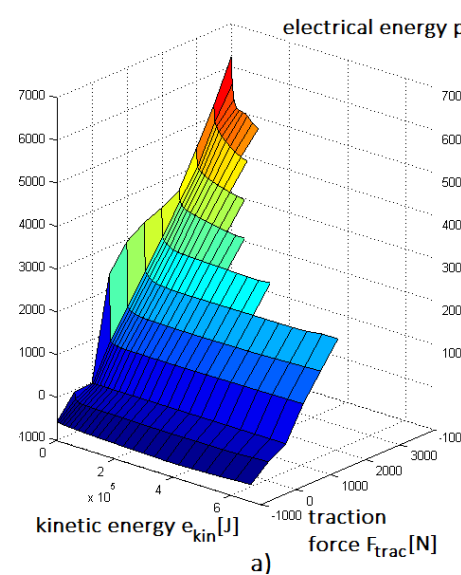

a)

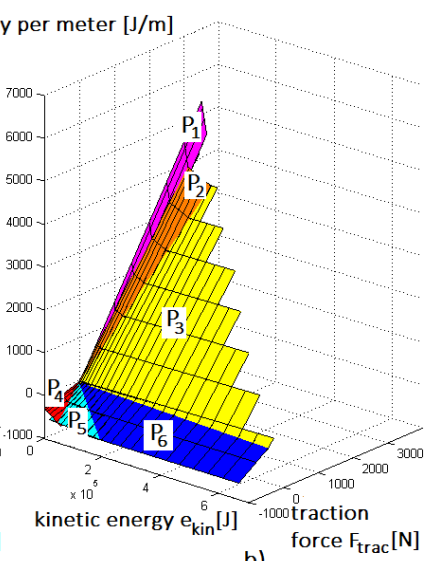

b)

Fig. 4. The figure shows the vehicle energy consumption per meter. The $x-$ axis has been rescaled in terms of the kinetic energy of the moving vehicle. a) gives the measured characteristics; b) gives the approximation by six linear functions.

single lower order polynomial. The use of a piecewise linear problem formulation would be appropriate but in general requires the use of different dynamic models in different regions of the state space (i.e. operating points) which makes the problem more time-consuming to solve. This can be avoided by using a convex piecewise linear approximation. Convex piecewise linear functions can be represented by the maximum of a set of linear functions. This maximum can be determined implicitly in the optimisation problem with no need for a special solver.

To achieve this, (in this case) six linear functions ( $P_{1}$ to $P_{6}$ ) are introduced that form the lower boundary of a convex set. They are fitted to the different regions of the map in Fig. 4a by a least-squares regression, see Fig. 4b. Secondly, $P_{1}$ to $P_{6}$ are transformed into inequality constraints on a decision variable $u_{\text {cons }}$ that represents the energy consumption of the vehicle per meter. The constraints have the form:

$$
P_{i}: \quad u_{\text {cons }} \geq a_{i} \cdot e_{k i n}+b_{i} \cdot F_{\text {trac }} \quad \mathrm{i}=1 \ldots 6
$$

The optimisation problem (as discussed later in Section III-B) is then set up in a way that guarantees that one of these constraints is always equally fulfilled by the decision variable $u_{\text {cons }}$ and it hence represents the energy consumption per meter of the vehicle according to the following piecewise linear model:

$$
\begin{array}{ll}
u_{\text {cons }}=a_{i} \cdot e_{k i n}+b_{i} \cdot F_{\text {trac }} & \text { if } P_{i} \text { is active } \\
u_{\text {cons }} \geq a_{j} \cdot e_{\text {kin }}+b_{j} \cdot F_{\text {trac }} & \text { for } j \neq i
\end{array}
$$

In other words, it can be stated that one of the inequality constraints (11) is always active depending on the actual operating point specified by the speed $v$ and the traction force $F_{\text {trac }}$. Hence, the variable $u_{\text {cons }}$ represents a piecewise linear approximation of the power consumption per meter without the necessity of defining a piece-wise changing dynamic model explicitly and using a solver for piece-wise linear problems.
Given this information, the energy consumption of the vehicle $E_{e l}$ can simply be computed by integrating the decision variable $u_{\text {cons }}$ with respect to the position.

$$
\frac{d E_{e l}}{d s}=u_{\text {cons }}(s)
$$

Approximating nonlinear maps by the maximum of linear functions is a known technique in nonlinear optimisation and called separable programming [14]. However, to the best of the authors' knowledge, this method has so far not been used to model piecewise linear dynamics in MPC formulations.

3) Model Discretisation: The continuous dynamic motion model (8) and the model of the energy consumption (13) can be summarised in state space form. Once a sampling step $\Delta s$ is chosen, the linear dynamic model can be discretised in a straightforward way assuming zero-order hold for the input and the disturbance to obtain the following discrete state space representation for $k \in\{0 \ldots . N-1\}$, where the "hat" symbolizes the discrete counterparts of the continuous variables:

$$
\begin{array}{r}
\underbrace{\left[\begin{array}{c}
\hat{e}_{k i n, k+1} \\
\hat{E}_{e l, k+1}
\end{array}\right]}_{\hat{x}_{d, k+1}}=\underbrace{\left[\begin{array}{cc}
a_{11} & 0 \\
0 & 1
\end{array}\right]}_{A_{d}} \cdot \underbrace{\left[\begin{array}{c}
\hat{e}_{k i n, k} \\
\hat{E}_{e l, k}
\end{array}\right]}_{\hat{x}_{d, k}}+\underbrace{\left[\begin{array}{cc}
b_{11} & 0 \\
0 & \Delta s
\end{array}\right]}_{B_{d}} \cdot \underbrace{\left[\begin{array}{c}
\hat{F}_{\text {trac }, k} \\
\hat{u}_{\text {cons }, k}
\end{array}\right]}_{\hat{u}_{d, k}} \\
+\underbrace{\left[\begin{array}{cc}
e_{11} & e_{12} \\
0 & 0
\end{array}\right]}_{E_{d}} \cdot \underbrace{\left[\begin{array}{c}
\sin \left(\hat{\alpha}_{s l, k}\right) \\
\cos \left(\hat{\alpha}_{s l, k}\right)
\end{array}\right]}_{\hat{d}_{d, k}}
\end{array}
$$

\section{B. Overall Problem Formulation}

Based on the results of the previous sections, the complete model-predictive eco-cruise control problem is formulated as a discrete finite-dimensional optimisation problem with a quadratic cost function and linear constraints. The cost function includes the energy consumption at the last step of the prediction horizon to achieve an "intelligent" predictive controller behaviour with the freedom to increase the consumption at any position if there is the benefit to save more energy later as a result of this anticipatory action:

$$
M_{1}=Q_{1} \cdot \hat{E}_{e l, N}=Q_{1} \cdot \sum_{k=0}^{N-1} \hat{u}_{c o n s, k} \cdot \Delta s
$$

The kinetic energy tracking error at the last step of the prediction horizon is included in the cost function in order to prevent the controller from planning an undesirable standstill of the vehicle at the end of the prediction horizon:

$$
M_{2}=Q_{2} \cdot\left(\hat{e}_{k i n, N}-\hat{e}_{k i n, r e f, N}\right)^{2}
$$

The accumulated kinetic energy tracking error finally is a measure for the deviation from the speed reference trajectory:

$$
L=Q_{3} \cdot \sum_{k=1}^{N}\left(\hat{e}_{k i n, k}-\hat{e}_{k i n, r e f, k}\right)^{2}
$$

The complete optimisation problem is then given as follows: 


$$
\min _{\hat{F}_{\text {trac }}, \hat{u}_{\text {cons }}} M_{1}+M_{2}+L
$$

subject to the model of the system dynamics:

$$
\hat{x}_{d, k+1}=A_{d} \cdot \hat{x}_{d, k}+B_{d} \cdot \hat{u}_{d, k}+E_{d} \cdot \hat{d}_{d, k}
$$

subject to the initial conditions:

$$
\hat{E}_{e l, 0}=E_{e l}(0) ; \quad \hat{e}_{k i n, 0}=e_{k i n}(0)
$$

subject to the limitations of the traction force:

$$
0 \leq \hat{e}_{k i n, k} \quad F_{t r, \text { min }} \leq \hat{F}_{t r a c, k} \leq c_{1} \cdot \hat{e}_{k i n, k}+c_{2}
$$

subject to the approximations of the power consumption map:

$$
\hat{u}_{c o n s, k} \geq a_{i} \cdot \hat{e}_{k i n, k}+b_{i} \cdot \hat{F}_{\text {trac }, k} \quad \mathrm{i}=1 \ldots 6
$$

Since problem (18) only consists of linear and quadratic cost function terms as well as linear constraints, it can be rewritten in the standard form of quadratic programming with the optimisation variables $y$ and the weighting matrices $Q_{w}$ and $h_{w}$ :

$$
\min _{y} \frac{1}{2} \cdot y^{T} Q_{w} y+h_{w}^{T} \cdot y \quad \text { s.t. } \quad A_{c o n} \cdot y \leq b_{c o n}
$$

If $Q_{w}$ in problem (19) is positive definite, the quadratic program is strictly convex. The transformation of problem (18) into the form of (19) and the proof of convexity are omitted here for brevity.

As already mentioned in Section III-A.2, the problem formulation (18) includes a piecewise linear model of the decision variable $\hat{u}_{\text {cons }}$ specified by the inequality constraints (18e). This is proven in the sequel.

Proposition 1: Consider an optimisation problem with the following properties:

- Property 1: The cost function terms are separable with regard to the vector optimisation variables $u$ and $w$ with the elements $u_{k}, w_{k}$, where $k \in\{0 \ldots N-1\}$ :

$$
\min _{u, w} f(u)+g(w)
$$

- Property 2: The decision variable $u$ is subject to convex lower bounds:

$$
\mathcal{P}: \quad A \cdot\left[\begin{array}{l}
u \\
w
\end{array}\right] \geq b
$$

- Property 3: $f(u)$ is strictly monotonously decreasing for all $u_{k}, \quad k \in\{0 \ldots N-1\}$ in the feasible region: $f\left(u_{1}\right)<f\left(u_{2}\right) \quad \forall u_{1, k}, u_{2, k}: u_{1, k}<u_{2, k}$

Let $\left\{u^{*}, w^{*}\right\}$ be the solution of this optimisation problem. Then, $u^{*}$ will lie on the boundary of the feasible region, i.e. at least one of the inequality constraints will be equally fulfilled /active.

Proof: Suppose, for the sake of contradiction, that for a feasible pair $\{u, w\}$ no inequality constraint is active, i.e. $A \cdot\left[\begin{array}{c}u \\ w\end{array}\right]>b$.

Then, the value of the cost function could be reduced because there exists a vector $u^{\prime} \neq u$ where $u_{k}^{\prime} \leq u_{k} \quad \forall k \in$ $\{0 \ldots N-1\}$ such that $f\left(u^{\prime}\right)+g(w)<f(u)+g(w)$ and

$$
A \cdot\left[\begin{array}{l}
u^{\prime} \\
w
\end{array}\right]=b
$$

This follows from Property 3 and the fact that the constraint set on $u$ is lower bounded and convex. Thus, the pair $\{u, w\}$ cannot be the minimal solution. Hence, the solution must always lie on the boundary of the feasible set and at least one inequality constraint $P_{i} \in \mathcal{P}$ must be equally fulfilled.

Corollary 1: The solution $\hat{u}_{\text {cons }}^{*}$ of the proposed optimal control problem (18) always lies on the boundary of the feasible region and thus contains a piecewise linear model of $\hat{u}_{\text {cons }}$ according to its lower limits (18e).

Proof: The proof consists of showing that Properties 1-3 of Proposition 1 hold for the proposed problem (18).

Property 1 is fulfilled for the assignment $u=\hat{u}_{\text {cons }}$

$$
\begin{aligned}
& f(u)=M_{1}=Q_{1} \cdot \hat{E}_{e l, N}=\sum_{k=0}^{N-1} \hat{u}_{c o n s, k} \cdot \Delta s \\
& g(w)=M_{2}+L
\end{aligned}
$$

since the cost function terms $M_{2}$ and $L$ and thus $g(w)$ are independent of $\hat{u}_{\text {cons }}$.

Property 2 is fulfilled because the inequality constraints (18d) and (18e) form a convex and lower bounded set by definition according to Section III-A.2, Fig. 2 and Fig. 4 b.

Property 3 is fulfilled because a reduction of $\hat{u}_{c o n s, k}$ at any step $k$ always leads to a reduction of the energy consumption at the end of the prediction $\hat{E}_{e l, N}$. Hence, $M_{1}$ is strictly monotonously decreasing with the decision variable $\hat{u}_{c o n s, k}$.

Thus, $\hat{u}_{\text {cons }}$ will lie on the boundary of the feasible region defined by the inequality constraints (18e).

\section{Simulation of the Closed-Loop Control}

For the simulation of the control loop, the proposed MPC controller is tested with the nonlinear dynamic motion model formulated in terms of time (4) and the measured vehicle energy consumption (Fig. 3). The traction force is limited according to the measured curves in Fig. 2. A scenario including down-hill and up-hill driving is chosen according to Fig. 5a+b. The prediction horizon of the MPC controller is subdivided into 40 steps of $10 \mathrm{~m}$. This scenario is simulated twice - once with the proposed eco-cruise controller and once with the same MPC controller but with a zero weight on the energy consumption (pure kinetic energy reference tracking) for comparison. The results of the described scenario are computed within SIMULINK and depicted in Fig. 5. The weightings $Q_{1}$ to $Q_{3}$ as well as the prediction horizon length are tuned manually here and will be investigated more systematically in further research.

The eco-cruise controller accelerates to a constant steady driving speed of $64.1 \mathrm{~km} / \mathrm{h}$ on the even road segment. Before the down-slope is reached, the vehicle decelerates. This shows the predictive behaviour of the MPC controller and 
TABLE I

PARAMETERS OF THE CONTROLLER SETUP

\begin{tabular}{|l|l||l|l||l|l|}
\hline Symbol & value & symbol & value & symbol & value \\
\hline$A_{v}$ & $1.95 \mathrm{~m}^{2}$ & $m_{e q}$ & $1070 \mathrm{~kg}$ & $c_{d}$ & 0.37 \\
\hline$m_{l}$ & $160 \mathrm{~kg}$ & $c_{r}$ & 0.01 & $m_{v}$ & $900 \mathrm{~kg}$ \\
\hline$g$ & $9.81 \frac{\mathrm{m}}{\mathrm{s}^{2}}$ & $\rho_{a}$ & $1.2 \frac{\mathrm{kg}}{\mathrm{m}^{3}}$ & $F_{t r, \text { min }}$ & $-658 \mathrm{~N}$ \\
\hline$a_{1}$ & -0.0423 & $a_{2}$ & -0.0034 & $a_{3}$ & $1.27 \mathrm{E}-4$ \\
\hline$a_{4}$ & -0.0054 & $a_{5}$ & $-5.9 \mathrm{E}-4$ & $a_{6}$ & $5.64 \mathrm{E}-6$ \\
\hline$b_{1}$ & 1.5274 & $b_{2}$ & 1.3390 & $b_{3}$ & 1.2307 \\
\hline$b_{4}$ & 0.2876 & $b_{5}$ & 0.5048 & $b_{6}$ & 0.62 \\
\hline$c_{1}$ & -0.0056 & $c_{2}$ & 3505 & $Q_{1}$ & $1.45 \mathrm{e} 6$ \\
\hline$Q_{2}$ & 5 & $Q_{3}$ & 0.5 & & \\
\hline
\end{tabular}

a)
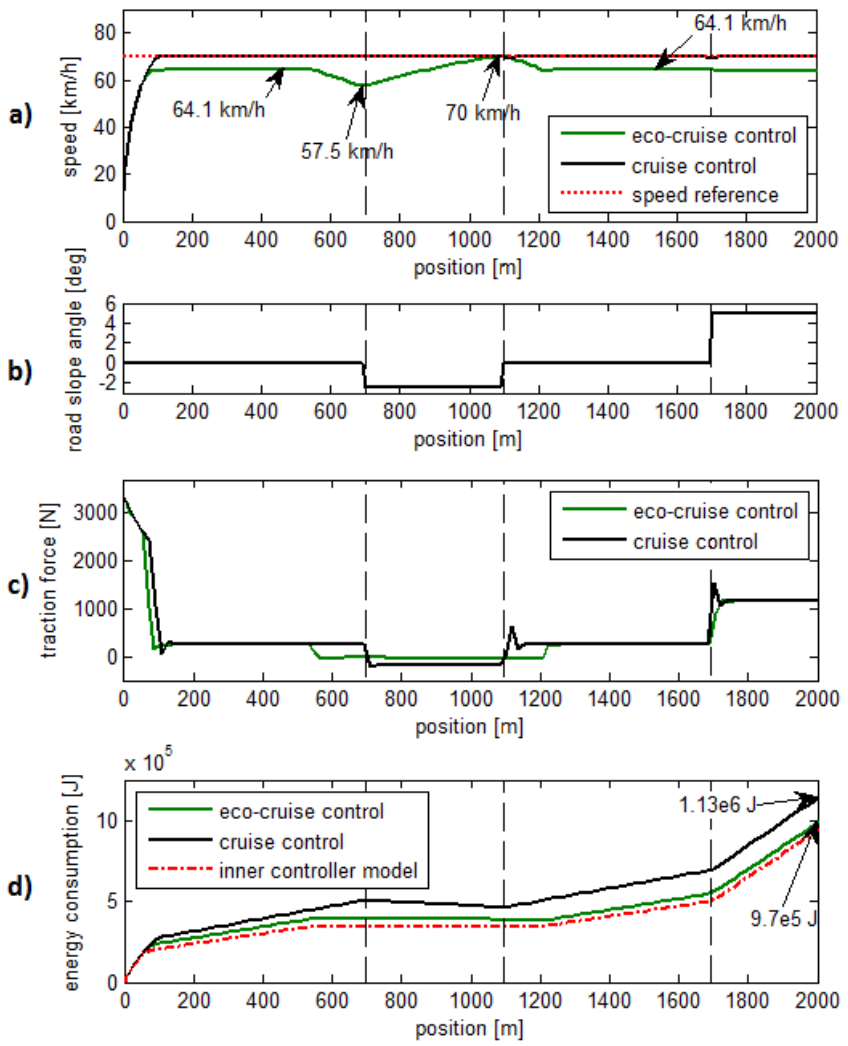

Fig. 5. Simulation results of the MPC controller in closed-loop operation.

serves to save energy since the speed loss can be recovered with no traction force effort during the upcoming downslope. At down-hill driving, the vehicle accelerates up to the desired speed of $70 \mathrm{~km} / \mathrm{h}$. This kinetic energy reserve allows to save energy on the following even road segment. In front of the following up-slope, the driving speed is decreased again down to $64.1 \mathrm{~km} / \mathrm{h}$.

This eco-cruise control strategy reduces the overall energy consumption throughout the simulation by $14.2 \%$ compared to the pure reference tracking controller while the average speed drops by only $8.2 \%$ which is a remarkable result.

Finally, Fig. 5d shows the comparison between the energy consumption computed with the inner controller model (Fig. 4b) and the measured characteristic map (Fig. 3) in ecocruise control which shows a good accordance.

Convex quadratic programs can be solved in polynomial time which is a good basis for a real-time capable algorithm. In practical tests, the optimisation shall be solved every 0.1 seconds. The computational time to solve the optimisation problem within MATLAB on a desktop PC (Intel Core i7) varies between 0.05 and 0.15 seconds during the presented simulation. Solving the same problem with a C-code based quadratic programming solver, a significantly faster computation is expected. Thus, the proposed problem formulation is expected to be real-time capable.

\section{CONCLUSION}

The eco-cruise control problem is converted into the form of a quadratic optimisation with linear constraints without applying an overall linearisation. The motion equation is reformulated to obtain a linear differential equation. The energy consumption of the vehicle is modeled by the maximum of a set of linear functions that is determined implicitly by the optimisation which makes the formulation equivalent to the use of a piecewise linear model. This provides a better fit of the vehicle characteristics than lower order polynomials. The proposed formulation guarantees a fast solution and is much more suitable for a real-time implementation than a nonlinear problem formulation. The next steps will be a further investigation of the closed-loop performance and the practical implementation in the real vehicle.

\section{REFERENCES}

[1] J. N. Barkenbus, "Eco-driving: An overlooked climate change initiative," Energy Policy, vol. 38, no. 2, pp. 762 - 769, 2010.

[2] A. Schwarzkopf and R. Leipnik, "Control of highway vehicles for minimum fuel consumption over varying terrain," Transportation Research, vol. 11, no. 4, pp. 279 - 286, 1977

[3] B. Saerens, "Optimal control based eco-driving - theoretical approach and practical applications," Ph.D. dissertation, KU Leuven, 2012.

[4] F. Lattemann, K. Neiss, S. Terwen, , and T. Connolly, "The predictive cruise control a system to reduce fuel consumption of heavy duty trucks," SAE, Tech. Rep. 2004-01-2616, 2004.

[5] M. A. S. Kamal, M. Mukai, J. Murata, and T. Kawabe, "Model predictive control of vehicles on urban roads for improved fuel economy," Control Systems Technology, IEEE Transactions on, vol. 21, no. 3, pp. 831-841, 2013.

[6] E. Hellstroem, "Look-ahead control of heavy vehicles," Ph.D. dissertation, Linköping University, 2010

[7] T. Schwickart, H. Voos, J. R. Hadji-Minaglou, and M. Darouach, "An efficient nonlinear model-predictive eco-cruise control for electric vehicles," Industrial Informatics, 2013. INDIN 2013. 11th IEEE International Conference on, pp. 311-316, jul. 2013.

[8] X. Li, Y. Chen, and J. Wang, "In-wheel motor electric ground vehicle energy management strategy for maximizing the travel distance," in American Control Conference (ACC), 2012, June 2012, pp. 49934998.

[9] C. Kirches, "Fast numerical methods for mixed-integer nonlinear model-predicitve control," Ph.D. dissertation, Ruprecht-KarlsUniversität Heidelberg, 2010.

[10] L. Guzzella and A. Sciarretta, Vehicle Propulsion Systems - Introduction to Modeling and Optimization. Springer, 2013.

[11] Smart, "Specifications," October 2013. [Online]. Available: http://www.smart.de/

[12] N. Kohut, K. Hedrick, and F. Borrelli, "Integrating traffic data and model predictive control to improve fuel economy," 12th IFAC Symposium on Control in Transportation Systems, pp. 155-160, 2009.

[13] B. Saerens, H. Rakha, M. Diehl, and E. V. den Bulck, "A methodology for assessing eco-cruise control for passenger vehicles," Transportation Research Part D: Transport and Environment, vol. 19, no. 0, pp. 20 $-27,2013$

[14] S. Stefanov, Separable Programming - Theory and Methods. Kluwer Academic Publishers, 2001. 